Mellwig / Moxter / Ordelheide

Handelsbilanz und Steuerbilanz 
Frankfurter betriebswirtschaftliches Forum an der Johann Wolfgang Goethe-Universität

Professor Dr. Winfried Mellwig

Professor Dr. Adolf Moxter

Professor Dr. Dieter Ordelheide

Band 2 
Winfried Mellwig, Adolf Moxter, Dieter Ordelheide (Hrsg.)

\section{Handelsbilanz \\ und Steuerbilanz}

Beiträge zum neuen Bilanzrecht, Band 2

\section{GABLER}


CIP-Titelaufnahme der Deutschen Bibliothek

Beiträge zum neuen Bilanzrecht / Winfried Mellwig ... (Hrsg.). - Wiesbaden : Gabler.

(Frankfurter Betriebswirtschaftliches Forum an der Johann-Wolfgang-Goethe-Universität ; ...) NE: Mellwig, Winfried [Hrsg.]

Bd. 2. Handelsbilanz und Steuerbilanz. - 1989

Handelsbilanz und Steuerbilanz / Winfried Mellwig ... (Hrsg.). - Wiesbaden : Gabler, 1989. (Beiträge zum neuen Bilanzrecht ; Bd. 2)

(Frankfurter Betriebswirtschaftliches Forum an der Johann-Wolfgang-Goethe-Universität ; Bd. 2)

NE: Mellwig, Winfried [Hrsg.]; Frankfurter Betriebswirtschaftliches Forum: Frankfurter Betriebswirtschaftliches Forum ...

Der Gabler Verlag ist ein Unternehmen der Verlagsgruppe Bertelsmann

(C) Betriebswirtschaftlicher Verlag Dr. Th. Gabler GmbH, Wiesbaden 1989

Softcover reprint of the hardcover lst edition 1989

Lektorat: Ute Arentzen

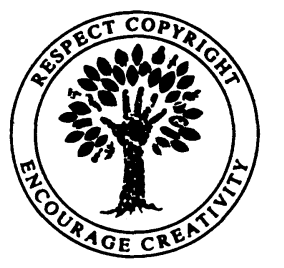

Das Werk einschließlich aller seiner Teile ist urheberrechtlich geschützt. Jede Verwertung außerhalb der engen Grenzen des Urheberrechtsgesetzes ist ohne Zustimmung des Verlages unzulăssig und strafbar. Das gilt insbesondere für Vervielfältigungen, Übersetzungen, Mikroverfilmungen und die Einspeicherung und Verarbeitung in elektronischen Systemen.

Satz: Lichtsatz Michael Glaese GmbH, Hemsbach 


\section{Vorwort}

Der Band 2 der Schriftenreihe „Frankfurter betriebswirtschaftliches Forum“ enthält weitere Vorträge zum neuen Bilanzrecht, die an der Universität Frankfurt a. M. gehalten wurden. Gegenstand der Vorträge ist das Verhältnis von Handels- und Steuerbilanz; insofern wird die Thematik des Band 1 „Einzelabschluß und Konzernabschluß“ fortgeführt und abgerundet. Das Interesse an den Veranstaltungen war wiederum sehr breit, was Wunder, sprachen doch Heinrich Beisse, Herbert Biener, Hermann Clemm, Manfred Groh, Rudolf J. Niehus, Hans Reintges, Arndt Raupach, Viktor Sarrazin, Manfred Sarx, Adalbert Uelner, Lothar Woerner.

Finanzielle Zuwendungen der Wilhelm-Merton-Stiftung haben die Fortführung der Veranstaltungsreihe ermöglicht. Hierfür danken wir ebenso wie für die professionelle Arbeit des Betriebswirtschaftlichen Verlages Dr. Th. Gabler GmbH.

WINFRIEd MELLWIG

Adolf MoXTER

Dieter Ordelheide 


\section{Inhaltsverzeichnis}

Die Generalnorm des neuen Bilanzrechts und ihre steuerrechtliche Bedeutung Von Prof. Dr. h.c. Heinrich Beisse

Vorsitzender Richter am Bundesfinanzhof

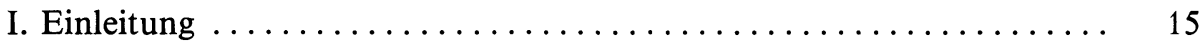

II. GoB-Generalnorm und „true and fair view“ $\ldots \ldots \ldots \ldots \ldots \ldots \ldots \ldots$

1. Konzeption des Gesetzgebers...................... 15

2. „True and fair view" - eine bilanzrechtliche Generalklausel? . . . . 17

3. Folgen einer solchen bilanzrechtlichen Generalklausel ......... 18

III. Die Generalnorm . . . . . . . . . . . . . . . . . . . . 19

1. Prämissen ................................. 19

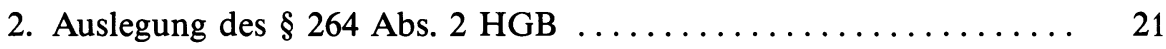

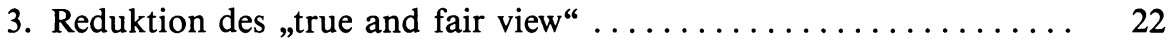

IV. Richtlinienkonformität $\ldots \ldots \ldots \ldots \ldots \ldots \ldots \ldots \ldots \ldots \ldots \ldots \ldots \ldots \ldots \ldots$

1. Fortwirkende Bedeutung der EG-Bilanzrichtlinie........... 23

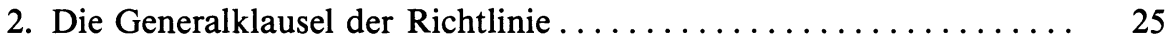

3. Korrekte Umsetzung der Richtlinie ................. 26

V. Steuerrechtliche Bedeutung der Generalnorm .............. 27

1. Kontinuität, Einheitlichkeit und Rechtssicherheit .......... 27

2. Weichenstellung: Maßgeblichkeitsgrundsatz .............. 28

3. Steuerneutralität des Bilanzrichtlinien-Gesetzes .............. 29

4. Rechtsprechungskompetenzen und Interpretationslast ........ 29

VI. Schluß: Europäisches Bilanzrecht $\ldots \ldots \ldots \ldots \ldots \ldots \ldots \ldots \ldots \ldots \ldots$

1. Zusammenfassung des Ergebnisses .................. 29

2. Gemeinschaftsrechtlicher Einfluß ................ 30

3. Vorwärtsstrategie: Europäisches Bilanzrecht ............ 30

Der schwebende Vertrag im Gefüge der Grundsätze ordnungsmäßiger Bilanzierung Vollständigkeitsgebot, Vorsichtsprinzip, Realisationsprinzip

Von Dr. Lothar Woerner

Vorsitzender Richter am Bundesfinanzhof

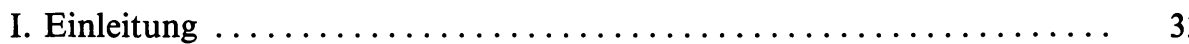




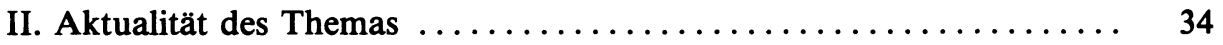

1. Rechtsprechung des Bundesfinanzhofs ............... 34

1.1. Das Urteil vom 27. 2. 1986 - IV R $52 / 83 \ldots \ldots \ldots \ldots \ldots \ldots . \ldots$

1.2. Das Urteil vom 29. 4. 1987 - IR $192 / 82 \ldots \ldots \ldots \ldots \ldots \ldots . \quad 35$

1.3. Analyse der Urteile ......................... 36

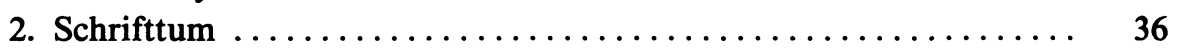

2.1. Gewinnrealisation beim Versendungskauf ............ 36

2.2. Gelhausen und die „Doppelbedeutung des Realisationsprin-

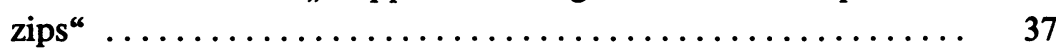

2.3. Analyse des Schrifttums ..................... 37

3. Strittige Problemkreise ......................... 38

III. Ausgangsfrage: Warum werden schwebende Geschäfte zunächst nicht bilanziert? .................................. 38

IV. Verhältnis von Vollständigkeitsgebot, Vorsichts- und Realisationsprinzip ........................... 40

1. Vollständigkeitsgebot und Vorsichtsprinzip .............. 40

2. Realisationsprinzip und Vollständigkeitsgebot $\ldots \ldots \ldots \ldots \ldots \ldots, 42$

3. Zusammenwirken mehrerer Bilanzierungsgrundsätze Zwischenergebnis............................. 43

V. Bedeutung des Schuldrechts für die Ermittlung des

Realisationszeitpunkts $\ldots \ldots \ldots \ldots \ldots \ldots \ldots \ldots \ldots \ldots \ldots \ldots \ldots \ldots \ldots \ldots, 44$

1. Vorrang des Bilanzrechts ...................... 44

2. Schuldrechtliche Ereignisse als Vermögenszuwachsindikatoren .... 46

3. Indikatoren des Schuldrechts - vier Thesen ............. 46

VI. Aufweis der Thesen an einzelnen Schuldverhältnissen ............ 48

1. Zweifelsfragen beim Kauf $\ldots \ldots \ldots \ldots \ldots \ldots \ldots \ldots \ldots, 48$

1.1. Kaufvertrag über bewegliche Sachen $\ldots \ldots \ldots \ldots \ldots \ldots \ldots$. 49

1.2. Kauf einer unbeweglichen Sache ................. 50

2. Spezialfall: Annahmeverzug $\ldots \ldots \ldots \ldots \ldots \ldots \ldots \ldots \ldots \ldots \ldots \ldots \ldots \ldots \ldots$

2.1. Streitiger Annahmeverzug ...................... 51

2.2. Unstreitiger Annahmeverzug .................... 51

3. Werkvertrag - Bedeutung der Abnahme des Werks.......... 52

4. Spielraum für einen „Dreipostenausweis“? .............. 53

5. Bedeutung von Gewährleistungsansprüchen, Unterschied zwischen Ansatz und Bewertung .......................... 54

VII. Schlußbemerkung $\ldots \ldots \ldots \ldots \ldots \ldots \ldots \ldots \ldots \ldots \ldots \ldots \ldots \ldots \ldots \ldots \ldots, \quad 55$ 
Die Scheingenauigkeit der steuerlichen Gewinnermittlung - Konsequenzen für Gesetzgebung, Verwaltung, Rechtsprechung und Steuerpflichtige

Von Dr. Hermann Clemm

Wirtschaftsprüfer, Rechtsanwalt und Steuerberater

I. Einleitung und Übersicht $\ldots \ldots \ldots \ldots \ldots \ldots \ldots \ldots \ldots \ldots \ldots$

II. Die Gründe der Scheingenauigkeit $\ldots \ldots \ldots \ldots \ldots \ldots \ldots \ldots \ldots$

1. Schätzungen und Prognosen ..................... 59

2. Die Benutzbarkeit der Bilanzierungsregeln (Gestaltungsmöglichkei-

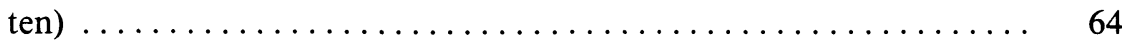

3. Zusammenfassung-Zwischenergebnis $\ldots \ldots \ldots \ldots \ldots \ldots \ldots 66$

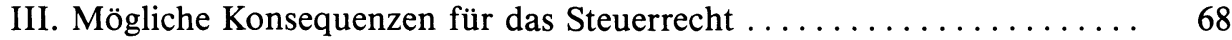

1. Der Gewinn als Besteuerungsschlüssel - historische Betrachtung .. 68

2. Abschaffung der Gewinnbesteuerung wegen Scheingenauigkeit und Gestaltbarkeit der Besteuerungsgrundlage Gewinn? - eine falsche Konsequenz

3. Der Gewinn als fragwürdiger, u. U. ungerechter, aber vertretbarer Schlüssel für die Besteuerung nach der Leistungsfähigkeit . . . . . . 70

4. Vertretbare Konsequenz: Die Verwendung der Handelsbilanz für

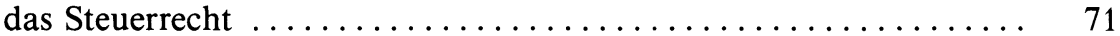

5. Überlegungen zu Steuerrecht und Steuermoral .............. 72

\section{Steuerliche Herstellungskosten und neues Handelsrecht}

Von Hans Reintges

Ehemaliges Mitglied des Vorstandes der Hoechst AG

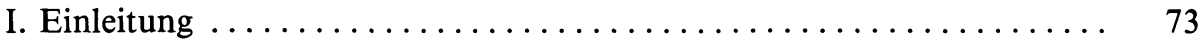

1. Systematik der gesetzlichen Regelung ................ 73

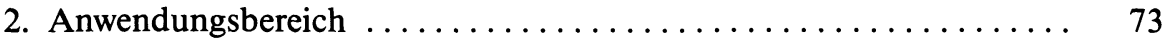

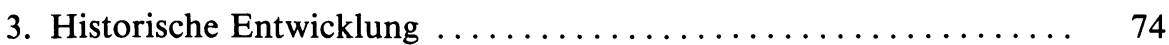

II. Definition und Bestandteile der Herstellungskosten im Handelsrecht . . 75

1. Allgemeiner Überblick . . . . . . . . . . . . . . . . . . 75

2. Aktivierungsfähige Gemeinkosten $\ldots \ldots \ldots \ldots \ldots \ldots \ldots \ldots \ldots$

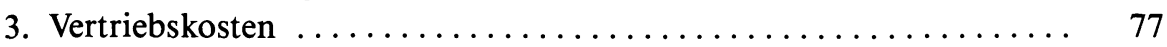

4. Aktivierungspflichtige Kosten und Herstellungskostenbegriff . . . . . 78

III. Bedeutung des handelsrechtlichen Herstellungskostenbegriffs für den

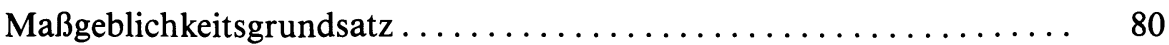

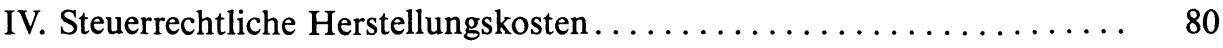

1. Vorschriften des Steuerrechts ..................... 80

2. Bedeutung der handelsrechtlichen GoB .............. 81 
3. Definition der Herstellungskosten in den EStR $\ldots \ldots \ldots \ldots \ldots \ldots \quad 82$

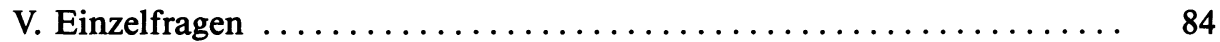

1. Forschungs- und Entwicklungskosten ................ 84

2. Bedeutung der Begriffe „angemessen“ und „notwendig“ ........ 84

3. Fremdkapitalzinsen $\ldots \ldots \ldots \ldots \ldots \ldots \ldots \ldots \ldots \ldots \ldots \ldots, 85$

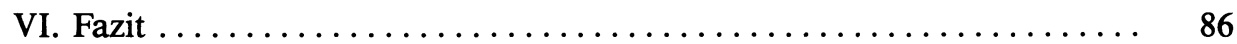

\section{Rückstellungen in Handels- und Steuerbilanz}

Von Dr. Adalbert Uelner

Ministerialdirektor im Bundesministerium der Finanzen

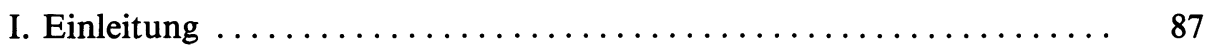

II. Zur Geschichte des Begriffs „Rückstellungen“ ............... 90

1. Rückstellungen im HGB vom 10. 5. $1887 \ldots \ldots \ldots \ldots \ldots \ldots \ldots .90$

2. Rückstellungen im Rahmen der Aktienrechtsnovelle von $1931 \ldots$... 90

3. Rückstellungen im Aktiengesetz von 1937 ................ 91

4. Rückstellungen im Aktiengesetz von $1965 \ldots \ldots \ldots \ldots \ldots \ldots . .61$

III. Rechtszustand nach dem Bilanzrichtlinien-Gesetz - § 249 HGB - . . 92

IV. Abgrenzungsregel bei Anwendung des $\S 249$ HGB $\ldots \ldots \ldots \ldots \ldots .93$

1. Grundlagen im Dritten Buch des HGB ................. 93

2. BFH-Rechtsprechung zur klaren Trennung zwischen Rückstellungen und Rücklagen.............................. 94

V. Die Voraussetzungen der Rückstellungsbildung im einzelnen ....... 96

1. Rückstellungen für ungewisse Verbindlichkeiten $\ldots \ldots \ldots \ldots \ldots .96$

1.1. Vorliegen einer Verbindlichkeit $\ldots \ldots \ldots \ldots \ldots \ldots \ldots \ldots .96$

1.2. Wirtschaftliche Verursachung $\ldots \ldots \ldots \ldots \ldots \ldots \ldots \ldots, \quad 97$

1.3. Inanspruchnahme des Verpflichteten ............... 98

1.4. Einfluß des $\S 12$ EStG ....................... 98

2. Rückstellungen für drohende Verluste aus schwebenden Geschäften 99

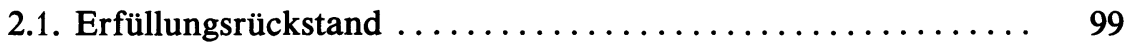

2.2. Drohender Verlust ........................ 99

3. Bewertung von Rückstellungen $\ldots \ldots \ldots \ldots \ldots \ldots \ldots \ldots \ldots \ldots, 100$

3.1. Rückstellungen für ungewisse Verbindlichkeiten.......... 100

3.2. Verluste aus schwebenden Geschäften .............. 101

VI. Aktuelle Rückstellungsfragen ........................ 101 


\section{Handelsbilanz und Steuerbilanz - Maßgeblichkeit und umgekehrte Maßgeblichkeit}

Von Prof. Dr. Arndt Raupach

Rechtsanwalt, Fachanwalt für Steuerrecht

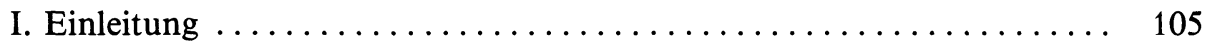

II. Zur Entwicklung des Maßgeblichkeitsgrundsatzes ............ 106

III. Die neuere Entwicklung zur umgekehrten Maßgeblichkeit . . . . . . . 108

1. Die Entscheidungen BFH I R 65/80 vom 24. 4. 1985 und BFH IV R 83/83 vom 25. 4. $1985 \ldots \ldots \ldots \ldots \ldots \ldots \ldots \ldots \ldots$

2. Einführung des $\S 6$ Abs. 3 EStG durch das BiRiLiG . . . . . . . 110

2.1. Eingeschränkter Geltungsbereich für Steuervergünstigungen . . 111

2.2. Keine Geltung für die Bildung steuerfreier Rücklagen ...... 111

2.3. Keine allgemeinen Grundsätze aus $\S 6$ Abs. 3 EStG ableitbar . . 112

IV. Die vorgesehenen Änderungen durch den Referentenentwurf für ein Steuerreformgesetz 1990 vom 14. 1. 1988 . . . . . . . . . . . . . . . . 113

V. Tragweite der vorgesehenen Verankerung der umgekehrten Maßgeb-

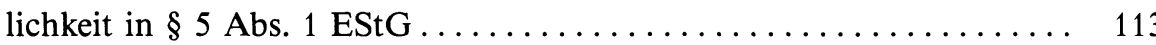

1. Was ist ein „steuerrechtliches Ansatz- und Bewertungswahlrecht"? . 114

2. Wann „entspricht" ein steuerrechtliches Bewertungswahlrecht einem handelsrechtlichen Wahlrecht?

3. Was bedeutet es, daß das Wahlrecht in der handelsrechtlichen Jahresbilanz ,ausgeübt" wird? ................... 115

4. Was ist eigentlich die maßgebende handelsrechtliche Jahresbilanz? . 115

VI. Bedenken gegen die umgekehrte Maßgeblichkeit.............. 116

\section{Abzinsung von Rückstellungen?}

Von Prof. Dr. Manfred Groh

Richter am Bundesfinanzhof

I. Rechtsprechung und Verwaltung ................... 119

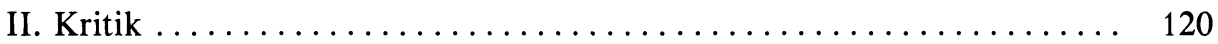

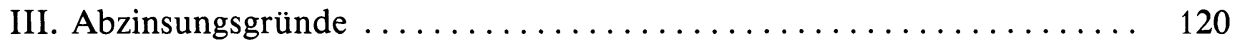

IV. Fingierte Zinsen in der BFH-Rechtsprechung? ............ 122

V. Folgen für Verbindlichkeitsrückstellungen $\ldots \ldots \ldots \ldots \ldots \ldots \ldots \ldots$

VI. Folgerungen für Verlustrückstellungen $\ldots \ldots \ldots \ldots \ldots \ldots \ldots \ldots \ldots$

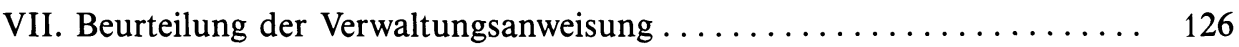


Ausgewählte Einzelprobleme der Bilanzierung

Von Prof. Dr. Manfred Sarx

Wirtschaftsprüfer und Steuerberater

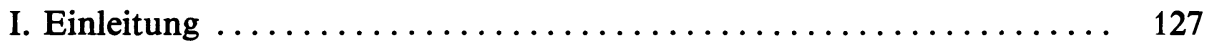

II. Sondereinzelkosten des Vertriebs $\ldots \ldots \ldots \ldots \ldots \ldots \ldots \ldots \ldots \ldots \ldots . \ldots \ldots$

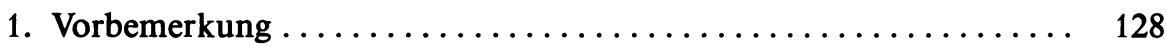

2. Aktivierungsverbot für Vertriebskosten ............... 130

3. Gegenwärtiger Meinungsstand .................... 130

4. Ergebnis in Thesenform ...................... 131

III. Teilgewinnrealisierung bei langfristiger Fertigung ........... 132

1. Vorbemerkung . . . . . . . . . . . . . . . . . . . . . . . . 132

2. Ausnahmen vom Realisationsprinzip ............... 133

3. Gegenwärtiger Meinungsstand $\ldots \ldots \ldots \ldots \ldots \ldots \ldots \ldots \ldots \ldots \ldots$

4. Sondereinzelkosten und Teilgewinnrealisierung $\ldots \ldots \ldots \ldots \ldots \ldots 135$

5. Ergebnis in Thesenform ...................... 135

IV. Sachverhaltsgestaltungen vor dem Bilanzstichtag und Generalklausel des $\S 264$ Abs. 2 HGB . . . . . . . . . . . . . . . . . . . . . . . . . 136

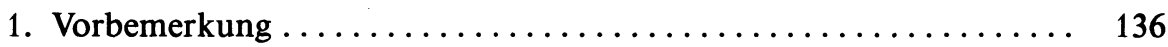

2. Allgemeine Grenzen der Sachverhaltsgestaltungen .......... 137

3. Auswirkungen der Generalnorm . . . . . . . . . . . . 138

V. Einzelprobleme des Fortführungsgrundsatzes .............. 139

1. Vorbemerkung ........................... 139

2. Inhalt und Grenzen des Fortführungsgrundsatzes .......... 140

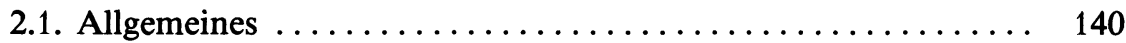

2.2. Grenzen der Fortführungsprämisse ............. 141

3. Rechnungslegung bei Wegfall der Fortführungsprämisse ........ 142

3.1. Allgemeines Bewertungskonzept ................ 142

3.2. Besonderheiten bei freiwilliger Auflösung . . . . . . . . . 143

3.3. Zur Anwendung der Generalklausel .............. 144

\section{Entwicklungen in der Gesetzgebung zum Maßgeblichkeitsgrundsatz}

Von Viktor Sarrazin

Ministerialrat im Bundesministerium der Finanzen

I. Vorbemerkung $\ldots \ldots \ldots \ldots \ldots \ldots \ldots \ldots \ldots \ldots \ldots \ldots \ldots \ldots \ldots \ldots$

II. Allgemeine Maßgeblichkeit . . . . . . . . . . . . . . . . 145

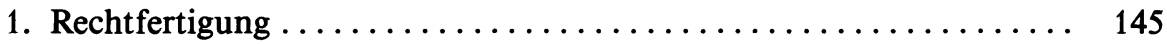

2. Bedeutung der Maßstabsregel $\ldots \ldots \ldots \ldots \ldots \ldots \ldots \ldots \ldots \ldots \ldots$ 
2.1. Ansatz einschließlich Wertansatz ............... 146

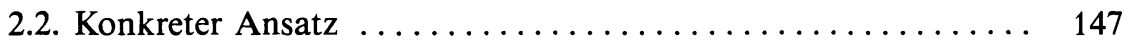

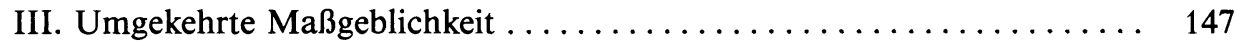

1. Begriff der umgekehrten Maßgeblichkeit . . . . . . . . . . 147

2. Umgekehrte Maßgeblichkeit als notwendige Folge der allgemeinen Maßgeblichkeit ........................... 149

3. Zweifel durch die neuere Rechtsprechung des Bundesfinanzhofs und die Reaktion des Gesetzgebers . . . . . . . . . . . . . 150

4. Streit um die Auslegung des $\S 6$ Abs. 3 EStG .............. 151

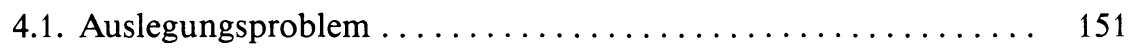

4.2. Die beiden Meinungen .................... 151

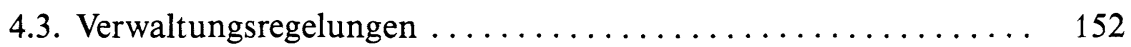

IV. Ergänzung des $\S 5$ Abs. 1 EStG durch eine Regelung zur umgekehrten Maßgeblichkeit ......................... 153

1. Vorschlag im Referentenentwurf eines Steuerreformgesetzes 1990 . . 153

2. Vorschlag im Regierungsentwurf eines Steuerreformgesetzes 1990 .. 154

3. Vorschlag von Mathiak ...................... 154

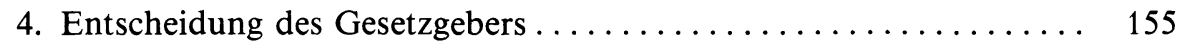

V. Bekenntnis des Gesetzgebers zum Maßgeblichkeitsgrundsatz . . . . . . 155

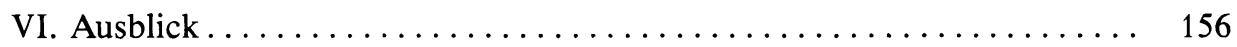

\section{Fragen zum Bilanzrichtlinien-Gesetz}

Von Herbert Biener

Ministerialrat im Bundesministerium der Justiz

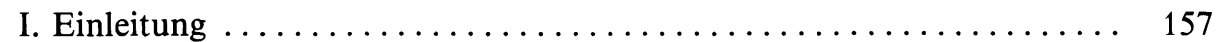

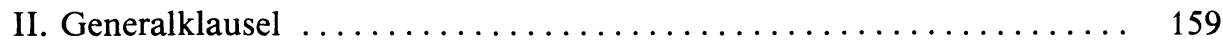

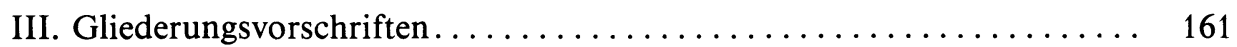

1. Allgemeine Anforderungen . . . . . . . . . . . . . . . . 161

2. Verlagerung von Angaben in den Anhang .............. 162

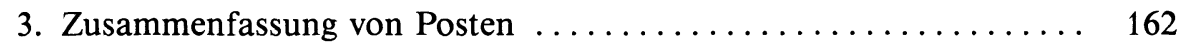

4. Ausweis der Rechnungsabgrenzung . . . . . . . . . . . . . . . . 164

5. Ausweis der erhaltenen Anzahlungen auf Bestellungen ........ 165

6. Ausweis der betrieblichen Steuern in der Gewinn- und

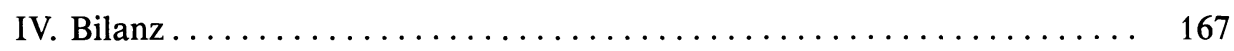

1. Verbundene Unternehmen ...................... 167

2. Latente Steuern ........................... 171

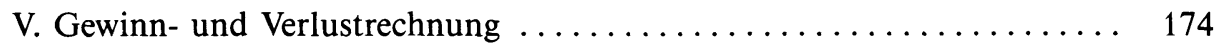

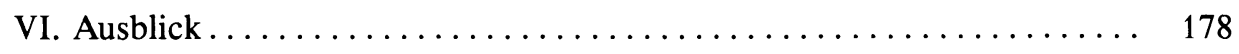


Der Anhang in nationaler und internationaler Betrachtung

Von Rudolf J. Niehus

Gesellschafter-Geschäftsführer der

Wollert-Elmendorff Deutsche Industrie-Treuhand GmbH

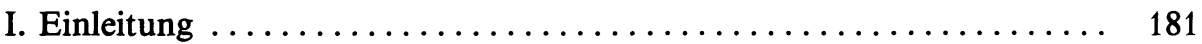

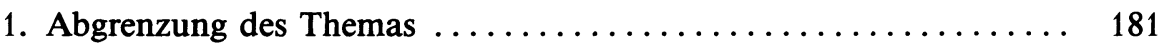

1.1. „Die Dinge sind im Fluß“ ................... 181

1.2. Keine vollständige Wiedergabe der Bestimmungen von $\S \S 284-288$ HGB $\ldots \ldots \ldots \ldots \ldots \ldots \ldots \ldots \ldots \ldots \ldots \ldots \ldots, 182$

1.3. „In internationaler Betrachtung“ ............... 182

2. Zur Genesis des Anhangs nach dem HGB ............... 184

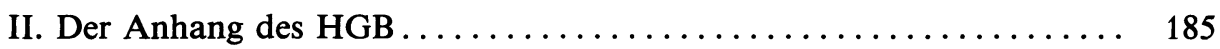

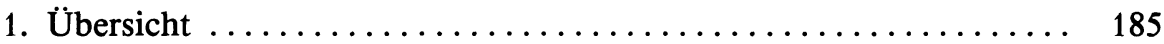

1.1. Bestandteile des Jahresabschlusses ............. 185

1.2. „Offener Anhang“ - Beschränkung auf gesetzliche Mindestregelungen ...................... 185

1.3. Verteilung über fünf Paragraphen des HGB . . . . . . . 186

2. Der Anhang des HGB als Reflex der EG-Harmonisierung ...... 186

2.1. Die Accounting Policies á la EG und international ........ 186

2.2. Die übrigen Regelungen $\ldots \ldots \ldots \ldots \ldots \ldots \ldots \ldots \ldots \ldots \ldots$

III. Die Funktionen $\ldots \ldots \ldots \ldots \ldots \ldots \ldots \ldots \ldots \ldots \ldots \ldots \ldots \ldots$

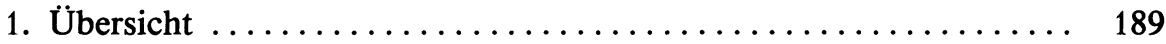

2. Interpretationsfunktion $\ldots \ldots \ldots \ldots \ldots \ldots \ldots \ldots \ldots \ldots \ldots \ldots \ldots \ldots$

2.1. Vermögenslage $\ldots \ldots \ldots \ldots \ldots \ldots \ldots \ldots \ldots \ldots \ldots \ldots \ldots \ldots$

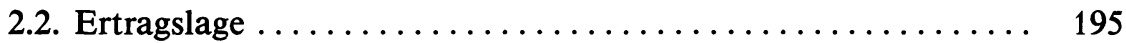

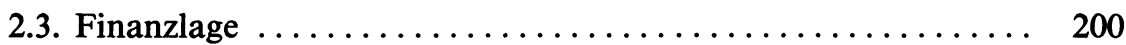

2.4. Zwischenergebnis ....................... 208

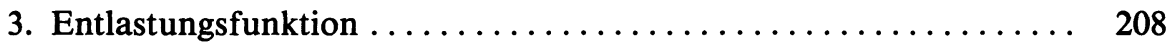

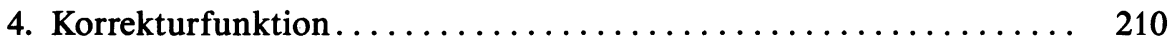

5. Allgemeine Zusatzinformationsfunktion ............. 211

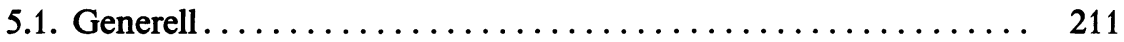

5.2. Die Informationsfunktion als Route zur Internationalität . . . 212

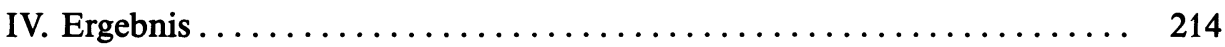

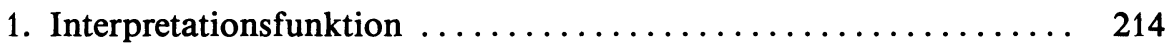

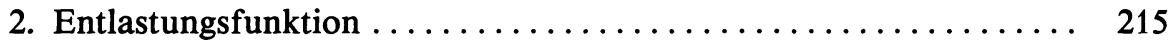

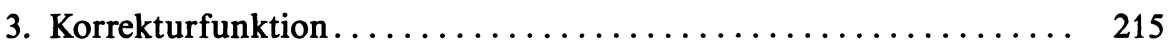

4. Informationsfunktion ..................... 215

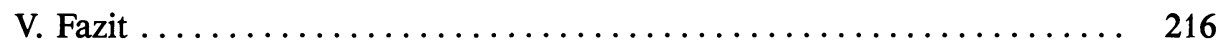

